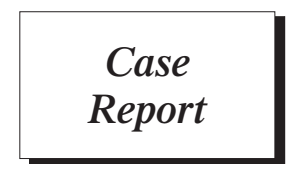

\title{
Percutaneous Cardiopulmonary Support of Video-Assisted Metastasectomy for a Patient with Lung Cancer Following Pneumonectomy
}

\author{
Toshiaki Suzuki, MD, Tadashi Akiba, MD, Miyako Hiramatsu, MD, Hideki Matsudaira, MD, \\ Jun Hirano, MD, Makoto Odaka, MD, and Toshiaki Morikawa, MD
}

\begin{abstract}
We describe herein a case of a 68-year-old woman with primary lung cancer who had undergone induction chemoradiotherapy and then a right pneumonectomy for non-small cell cancer (adenocarcinoma). Twenty-one months later, the cancer had metastasized to the brain, which was treated with 2-knife radiosurgery. She had been well for up to 32 months; however, the chest radiography and chest computed tomography (CT) demonstrated a nodule in the left upper lobe of the lung that was gradually growing. We preformed a partial resection of the left upper lobe by video-assisted thoracic surgery (VATS) under percutaneous cardiopulmonary support (PCPS), considering the oxygenation requirement of the patient. PCPS was applied via venoarterial ( $\mathrm{V}-\mathrm{A})$ bypass, and the hemodynamic status of the patient was mostly stable. The postoperative course was uneventful, and the patient has no evidence of metastatic lung cancer, 10 months postoperatively, indicating that the minimally invasive VATS under PCPS was successful and safe. Although few reports have been described and some key questions remain unanswered, the method appears to be promising.
\end{abstract}

Key words: lung, lung cancer, lung cancer surgery, percutaneous cardiopulmonary support, pneumonectomy

\section{Introduction}

Video-assisted thoracic surgery (VATS) is minimally invasive and may be used for patients with poor respiratory function. Percutaneous cardiopulmonary support (PCPS) is used for cardiac dysfunction and cardiopulmonary resuscitation. Here, we describe the usefulness of PCPS used during VATS in treating contralateral

$\overline{\text { Department of Thoracic Surgery, Tokyo Jikei University of }}$ Medicine, Tokyo, Japan

Received: June 16, 2009; Accepted: September 10, 2009

Corresponding author: Toshiaki Suzuki, MD. Department of General Thoracic Surgery, Tokyo Jikei University of Medicine, Jikei University Hospital, 163-1 Kashiwashita, Kashiwa, Chiba 277-8567, Japan

Email: toshiaki1129jp@yahoo.co.jp

(C)2011 The Editorial Committee of Annals of Thoracic and Cardiovascular Surgery. All rights reserved. pulmonary metastases in a patient who had a right pneumonectomy.

\section{Case}

A 68-year-old woman was diagnosed with primary lung cancer stage IIB in another hospital where she had undergone induction chemoradiotherapy and then a right pneumonectomy for non-small cell cancer (adenocarcinoma) (Fig. 1). Twenty-one months later, the cancer metastasized to the brain, which was treated by 2 -knife radiosurgery. She had been well for up to 32 months; however, the chest radiography and chest computed tomography (CT) demonstrated a nodule in the left upper lobe of the lung that was gradually growing. Although conservative treatment was recommended by the other hospital, she hoped to undergo surgery, so she was referred to Jikei University Hospital. Chest CT showed an irregular 


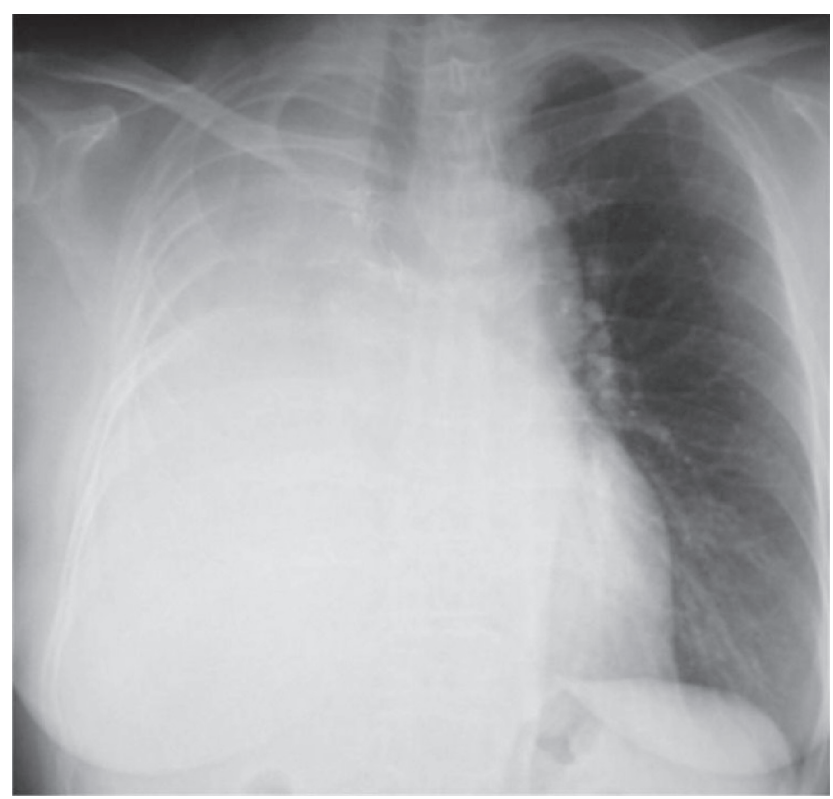

Fig. 1 Chest radiography showing right pneumonectomy.

coin lesion of $13 \times 13 \mathrm{~mm}$ in size in the superior segment $\left(\mathrm{S}_{3}\right)$ (Fig. 2). The lesion was diagnosed as metastatic lung cancer. The clinical test results for arterial blood gas analysis were $\mathrm{PaO}$ 2, 95.6 torr; and $\mathrm{PaCO}_{2}$, 41.6 torr under ambient conditions and test results for spirometry were FVC, $1240 \mathrm{ml}$; \%VC, $53.0 \%$; FEV ${ }_{1.0}, 980 \mathrm{ml}$. Since the general condition of the patient was good, we attempted the surgery, expecting to maintain the residual $\mathrm{FEV}_{1.0}$ at $900 \mathrm{ml}$ after surgery. The VATS partial resection was performed using PCPS under one-lung ventilation with 3 ports in the right lateral position. A total of $8000 \mathrm{U}$ of heparin was injected intravenously through a 17-Fr arterial cannula via the right femoral artery into the abdominal aorta and through a 21-Fr venous cannula via the right femoral vein into the right atrium, with a percutaneous cardiopulmonary support (PCPS) flow of $3.3 \mathrm{~L} /$ min. PCPS time was $75 \mathrm{~min}$ and the intraoperative hemodynamic status was stable. Oxygenation of the patient remained at $100 \%$ during operation with only minimal hand assist. The total bleeding volume was $85 \mathrm{ml}$, and she had no complications such as embolism, thrombosis, bleeding, or infection. The chest tube was removed on postoperative day 1 and the postoperative course was uneventful. The clinical test results of spirometry on postoperative day 5 were $\mathrm{FVC}, 1120 \mathrm{ml}$; $\% \mathrm{VC}, 47.9 \%$ and $\mathrm{FEV}_{1.0}, 1080 \mathrm{ml}$. The patient was discharged and remains well to date. Histopathological examination of the resected specimen showed metastatic carcinoma, and the surgical margin was negative for tumor.

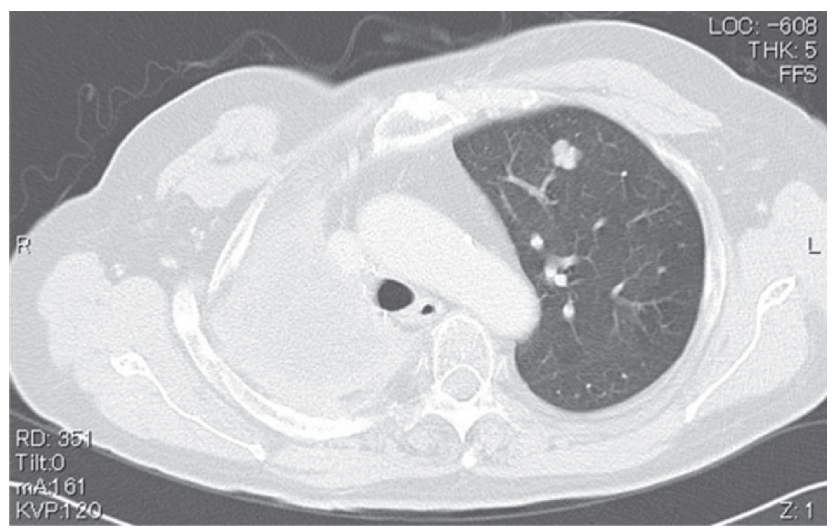

Fig. 2 Chest CT revealing carcinoma in the contralateral lung.

\section{Discussion}

Demmy ${ }^{1)}$ reported that the advantages of VATS lobectomy over traditional open surgical approaches remain controversial. Subjective concerns such as pain, dyspnea and physical functioning, particularly poor respiratory function, favor VATS. Tsubota ${ }^{2)}$, Kodama $^{3)}$ and Mizoguchi ${ }^{4)}$ reported that PCPS can contribute to hemodynamic stabilization, and so is useful for severe cardiac dysfunction, cardioplumonary resuscitation and particularly in the field of thoracic surgery, for cases of respiratory stenosis, central respiratory damage, bilateral pneumothorax or poor respiratory function. VATS demands lung collapse during surgical maneuvers, but in this case, if we had collapsed the lung, the patient would have suffocated. VATS under PCPS is thus a useful adaptation for cases of contralateral metastatic lung cancer after a pneumonectomy. Ishikawa ${ }^{5)}$ reported a case of contralateral pneumothorax after a pneumonectomy and described respiratory management of the patient using PCPS. However, few reports have examined VATS under PCPS for treating contralateral metastatic lung cancer after patients had undergone a pneumonectomy. We report herein the usefulness of VATS and PCPS for respiratory support in treating contralateral metastatic lung cancer after a pneumonectomy. During partial resection, oxygenation was maintained mostly at $100 \%$ with PCPS flow of $3.3 \mathrm{~L} / \mathrm{min}$ and jet ventilation. Despite preoperative heparinization with 8000 $\mathrm{U}$, bleeding was only $85 \mathrm{ml}$. Postoperative spirography showed no difference compared to preoperatively and no complications were encountered. The patient was discharged safely. Two methods are available for PCPS: venoarterial $(\mathrm{V}-\mathrm{A})$ bypass and venovenous $(\mathrm{V}-\mathrm{V})$ bypass: 
V-A bypass offers decreased preload volume to the heart; however, it has the disadvantage of providing an unequal supply of oxygen to the whole body; In contrast, V-V bypass provides an equal supply of oxygen to the whole body. ${ }^{6-8)}$ We selected V-A bypass because we predicted that decreased right pulmonary arterial circulation would result in increased right lung airway pressure and pulmonary vascular resistance, followed by a left lung collapse. Some controversial questions remain. First, we wondered whether PCPS really has any effect on the heart beat, as in this case. Intraoperatively, oxygen saturation dropped to $70-80 \%$ many times, so there is a possibility that the normal heart beat may have been acting in opposition to the PCPS circulation, which would mean that the oxygen saturation could not always be maintained. Second, with regard to brain circulation, although we used a V-A bypass, we could not be sure whether oxygenated blood really circulated into the brain, because oxygenated PCPS circulation to the brain might have been blocked by the cardiac circulation. Therefore, intracerebral oxygen saturation may need to be monitored.

\section{Conclusion}

We have described a case of contralateral metastatic lung cancer occurring after pneumonectomy that was successfully and safely treated using minimally invasive VATS under PCPS. Although few such reports have been described and some key questions remain unanswered, this method for pulmonary resection after pneumonectomy appears to be promising.

\section{References}

1) Demmy TL, Nwogu C. Is video-assisted thoracic surgery lobectomy better? Quality of Life Considerations. Ann Thorasic Surg 2008; 85: 719-28.

2) Tsubota N, Maeda M, Kameyama K, Sugita A, Hayashi $\mathrm{E}$, et al. Clinical experience with percutaneous cardiopulmonary support system (PCPS) for resection of pulmonary metastasis after pneumonectomy for lung cancer. J Thorac Cardiovasc Surg 1993; 7: 850-5.

3) Kodama K, Higashiyama M, Yokouchi H, Takami K, Yasuda T, et al. Use of percutaneous support (PCPS) for extended surgery in patients with T4 tumor. Kyobu Geka 2000; 53: 721-25.

4) Mizoguchi K, Saito R, Sinne S, Takahashi K. Anesthetic management for patients undergoing lobectomy using percutaneous cardiopulmonary support (PCPS). J Clin Anesth 2002; 26; 1121-2.

5) Ishikawa N, Saito H, Hiranuma C, Takizawa M. A surgical intervention using percutaneous cardiopulmonary support for contralateral pneumothorax following pneumonectomy. Ann Thorac Cardiovasc Surg 2001; 7: 235-7.

6) Tsunezuka Y, Sato H, Tsubota M, Seki M. Significance of percutaneous cardiopulmonary bypass support for volume reductuion surgery with severe hypercapnia. Artif Organs 2000; 24: 70-3.

7) Ishiyama $T$, Aoyama $T$, Hirahara H, Iwashima A, Tsukada $\mathrm{H}$, et al. Successful resection of endotracheal metastatic lung cancer using percutaneous cardiopulmonary support system. Kyobu Geka 2001; 54: 19-23.

8) Fukumori-Okamoto E, Nitahara K, Ikeda S, Yasumoto M, Higa K. Anesthetic management for partial lobectomy in a postpneumonectomy patient. J Clin Anesth 2005; $\mathbf{5 4} ; 1288-9$. 